\title{
Antioxidants Prevention of Diabetic Damage in the Organ Culture Bovine Lenses
}

\author{
E. Bormusov ${ }^{1 *}$, A. Dovrat ${ }^{2}$, M. Chevion ${ }^{2}$ and A. Z. Reznick ${ }^{1}$
}

${ }^{1}$ Rappaport Faculty of Medicine, Technion - Israel Institute of Technology, Haifa, Israel

${ }^{2}$ Faculties of Medicine and Dental Medicine, the Hebrew University of Jerusalem, Israel

\begin{abstract}
Oxidative stress represents a mechanism which could lead to diabetic cataract. We exposed bovine lenses in culture conditions for two weeks to high glucose concentration $(450 \mathrm{mg} \%)$ and investigated the damage to the lens and possible protection by special antioxidants - $\mathrm{N}$-acetyl-L-cysteine (NAC) and the zinc complex of desferrioxamine (DFO), a selective chelator for iron. We monitored the optical quality of the lenses and the oxidation of the epithelium with dichlorofluorescein (DCF) assay, as well as the changes in lens proteins profile by 2D gel electrophoresis. Under high glucose changes in lens focal length, increased oxidation, and changes in lens crystalline were observed. NAC and Zn- DFO nearly completely protected the lenses; DFO showed only partial protection. The results demonstrated that antioxidants should be considered as treatment modality protecting the lens from high glucose damage. It is proposed that a combination of NAC and Zn/DFO could prove highly efficient.
\end{abstract}

\section{Introduction}

Cataract is a highly prevalent complication in diabetes. Several mechanisms explaining the formation of diabetic cataract have been proposed, including those that involve oxidative stress, as a causative factor.

The "Free Radical Theory of Aging", first proposed in 1956 by Denham Harman [1] (reviewed by Harman 2003[2]), suggests that aging results from accumulation of changes caused by highly reactive oxygen-derived species (ROS), including free radicals, known to induce cell damage [3]. Continuous exposure of the lens to oxidative stress has been shown to lead to reduced lens clarity and cataract formation [4]. Since the epithelial layer contains the bulk of the metabolic enzymes, and since labile iron has been incriminated as a necessary factor for injurious oxidative stress, the damage to the epithelial layer can precede and account for the development of lens opacity [5]. Indeed, the levels of redox active iron was found elevated in the advanced forms of cataract [6].

Desferrioxiamine (Desferal $\left.{ }^{\circledR}, \mathrm{DFO}\right)$ is a selective high affinity iron chelator, rendering iron as a stable ferric complex [7]. DFO has been often used as a means of reducing tissue oxidative stress and injury. Reddan et al. demonstrated that DFO protects cultured rabbit lens epithelial cell from oxidative insult [8]. Avunduk et al. showed the effectiveness of DFO in preventing cataractous changes in rat lenses following in vivo exposure of rats to oxidative stress [7]. We prepared the complex of DFO with zinc and gallium [9-13]. These complexes are similar to the ferrioxamine (Fe(III)-DFO complex), but show three additional advantages: (i) they better infiltrate into cells, (ii) are less toxic than DFO alone, and (iii) act via the combination of both 'push' and 'pull' mechanisms. Thus, we examined the protective effect of these DFO complexes on cataract formation in bovine lens, under conditions simulating the diabetic state.

We have used an additional alternative strategy for curbing ROSinduced injury and protection of the lens. This involved the employment of N-acetyl cysteine (NAC), to scavenge free radicals [14-16] and to replenish reduced sulfhydryl residues [14-19]. NAC is a precursor of glutathione - the major source of cellular sulfhydryl groups and acts as a potent an anti-inflammatory agent.

Experimentally, we incubated intact bovine lenses in culture, in the presence of high glucose concentrations (450 $\mathrm{mg} \%$ ), mimicking the diabetic state, and examined the protection bestowed by the DFO complexes and NAC, on the injurious processes on the lenses. We used a unique system of intact bovine lenses, maintained for a longterm, under culture conditions. This system allows for direct exposure to a pre-set glucose concentration, and monitoring the effects on lens transparency with a highly sensitive optical method [20]. The system can detect early optical damage to lenses, which cannot be detected by other methods often used. At the completion of the culture period lens epithelium and lens proteins were analyzed.

It was anticipated that the extent of protection exerted on the lens, in this system, will be indicative of the involvement of iron as a catalyst and of free radicals as causative agents, in the damage to the diabetic lens.

\section{Materials and Methods}

\section{Lens organ culture system}

Lenses were excised in a delicate operation from eyes obtained from 1-year-old male calves under sterile conditions, 2-4 hours after enucleating. From each animal one eye is used for experimental treatment and the other eye serves as control. Each lens is placed in a specially designed culture container, which we have developed [21]. The culture medium consists of M199 with Earl's balanced salt solution, supplemented with 5.96g/L HEPES, 3\% dialyzed fetal calf serum and antibiotics (penicillin $100 \mathrm{U} / \mathrm{ml}$ and streptomycin $0.1 \mathrm{mg} /$ $\mathrm{ml})$. Our intact lens culture system mimics the lens conditions inside the eye and makes it possible to keep lenses for long-term studies for

*Corresponding author: Dr. Elvira Bormusov, Rappaport Faculty of Medicine, Technion - Israel Institute of Technology, Haifa, Israel, E-mail: bormusov@techunix.technion.ac.il

Received March 27, 2012; Accepted June 21, 2012; Published June 23, 2012

Citation: Bormusov E, Dovrat A, Chevion M, Reznick AZ (2012) Antioxidants Prevention of Diabetic Damage in the Organ Culture Bovine Lenses. Metabolomics S1:004. doi:10.4172/2153-0769.S1-004

Copyright: (c) 2012 Bormusov E, et al. This is an open-access article distributed under the terms of the Creative Commons Attribution License, which permits unrestricted use, distribution, and reproduction in any medium, provided the original author and source are credited. 
several weeks in order to test the effects of potentially damaging agents. The lenses were incubated at $35^{\circ} \mathrm{C}$

Experimental treatments were initiated after pre-incubation for 24 hours. Damaged lenses and their matched controls from the contra lateral eyes were excluded prior to experimental treatment. The culture medium is replaced every 24 hours.

\section{Lens optical quality monitoring system}

An automated scanning laser system [21] was used for daily testing of both treated and control lenses. A $670 \mathrm{~nm}$ diode laser with the beam parallel to the axis of the lens is directed towards the cultured lens along one meridian. After passing through the lens, the laser beam is refracted and the system determines the back vertex focal length for every beam position. Each scan consists of measurements of the same beam from 22 different points across the lens. A lens of good optical quality is able to focus the laser beam from various locations. When the lens is damaged, its ability to focus the laser beam at various locations is altered. High glucose concentration (simulating diabetes): Lenses were exposed to $450 \mathrm{mg} \%$ glucose in the culture medium, which simulates diabetes conditions. Preparation of lens epithelial samples for dichlorofluorescein (DCF) assay.

There were preparations of forward monolayer epithelium bovine lenses from all experiments. For this purpose the capsule opened and crystalline lens fibers were cleaned. On the object-plate here was only a capsule and a cellular monolayer epithelium.

\section{Reactive Oxygen Species (ROS)}

ROS was discovered by flow of epithelial cells lens labeled with 5-(and 6-)chloromethyl-2',7'-dichlorodihydrofluorescein diacetate, acetyl ester (CM- $\mathrm{H}_{2}$ DCFDA, C6827) to measure the component level of cellular oxidation in the cells of lens epithelium. When it is added oxidation to cells this reagent undergoes and converts in the fluorescent isomer. The fluorescent signal was detected with a fluorescence microscope, using sources of excitement and filters, corresponding to for fluorescein. Protein analysis using two-dimensional polyacrylamide gel electrophoresis was done according to [22]. Protein concentration was measured by the micro-method of Lowry [23]

\section{Results and Discussion}

The experiments included 78 intact bovine lenses, which were divided into eight different treatment groups. These groups include lenses incubated, for 12 days, with high glucose levels (450 mg\%) with or without each one of the antioxidants tested (NAC, Zn/DFO and $\mathrm{DFO}$ ), as well as control lenses. Lens optical quality was analyzed every 24 hours.

Figurel demonstrates the changes in the optical quality of the lenses along the incubation period. In the control group no significant change in the Back Vertex Distance (BVD) with time of incubation, was observed. Lenses incubated with high glucose showed fluctuations in the BVD along the incubation period, which indicate changes in lens volume. High fluctuations appear also in lenses treated with high glucose and NAC. The group exposed to ZnDFO and to DFO alone, demonstrated reduced optical changes representing smaller lens injury. The lenses show almost no volume changes.

Other studies also demonstrated changes in lens optical quality in diabetes [24] measured lens opacity in diabetic patients using a backlight scattering quantification system. Lens opacity was significantly higher in diabetic patients than in the control group, and showed correlation with glycated hemoglobin levels. Freel [25] compared cytoplasmic textures from a variety of human and animal lenses in electron microscope images in order to relate the extent of roughness with the extent of opacification. Lens cytoplasms exhibiting the greatest roughness correlated with the greatest light scattering. Tkachov [26] described histomorphological changes in the cataractous lens of diabetic patients using Scheimpflug densitometry and light microscopy. Their study revealed smaller cell density of the lens, larger cell area of lens epithelium, and a lower nucleus plasma ratio in cataractous lenses of diabetics compared to clear non-diabetic lenses.

In our study at the end of the culture period, lenses were photographed by inverted microscope. Figure 2a shows a photograph of a control lens with clear sutures and homogeneous epithelial cell layer. Figure $2 \mathrm{~b}$ demonstrates the severe damage to the lens, when incubated in the presence of high glucose, which is indicated by the blisters under and between the epithelial cells. Photographs of the lens epithelial layer with higher magnification (Figure 3) show that for the controls an intact lens epithelial layer is observed (Figure 3a). Glucose treated lenses show swollen epithelial cells with blisters at the cells borders (Figure $3 \mathrm{~b}$ ). Each of the three antioxidants used in the study reduces the glucose-induced damage to the lens epithelium (Figures $3 c-3 e)$.

The antioxidant protective effect of each of the three agents were

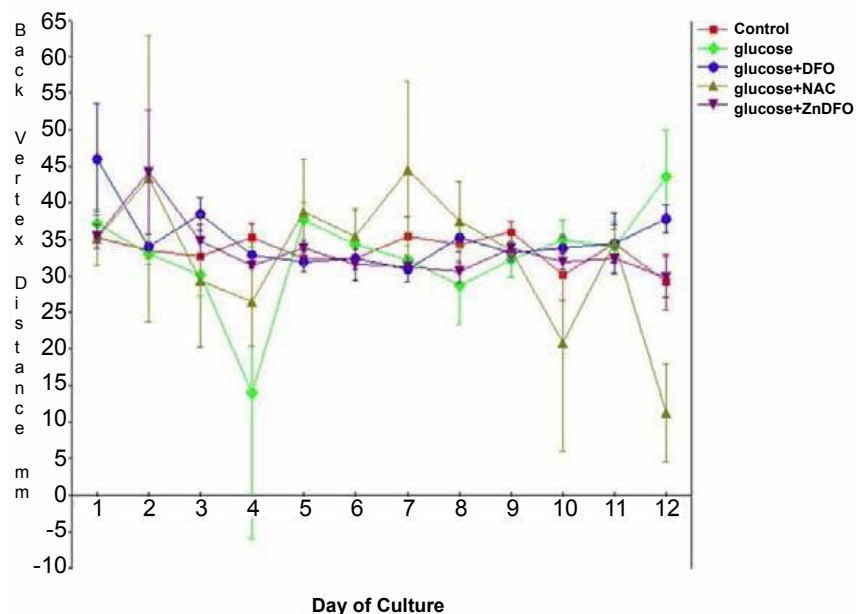

Figure 1: Lens optical quality during the twelve days of incubation of intact lenses in culture conditions, demonstrated by "Back Vertex Distance". Control lenses show almost no change in Back Vertex Distance with time in culture (red), while glucose treated lenses show variability in Back Vertex Distance which reflect changes in lens volume.

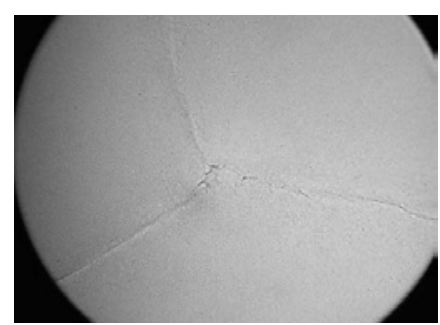

Figure 2a: Inverted Microscope photograph of control lens after 12 days incubation in organ culture conditions. (Magnification x25) Note the clear lens with lens sutures. 


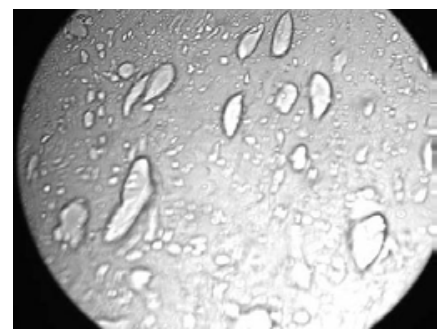

Figure 2b: Inverted Microscope photograph of glucose treated lens (450 $\mathrm{mg} \%)$, after 12 days incubation in organ culture conditions. The photo show high glucose damage as bubbles at the lens surface. (Magnification x25).

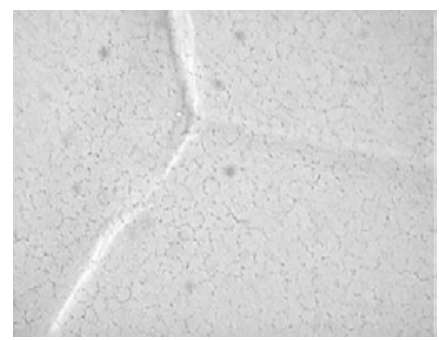

Figure 3a: Inverted microscope photograph of control lens epithelium after 12 days incubation in organ culture conditions. (Magnification $x 100$ ) Note the similar size of the cells and the borders between the cells.

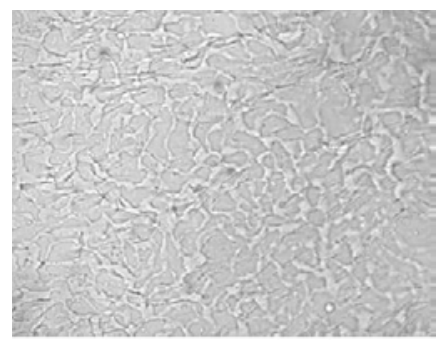

Figure 3b: Inverted microscope photograph of glucose treated lens epithelium after 12 days incubation in organ culture conditions. (Magnification $x 100$ ) note the swelling cells with different size and the bubbles between the cells.

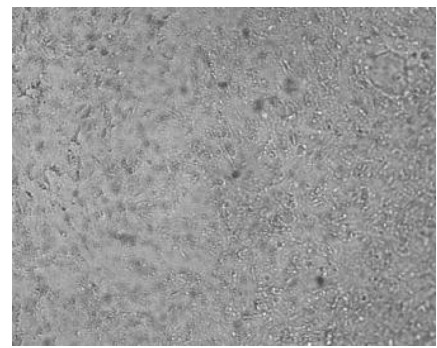

Figure 3c: Inverted microscope photograph of glucose and DFO treated lens epithelium after 12 days incubation in organ culture conditions. (Magnification $x$ 100) glucose damage was reduced by DFO treatment.

compared using the 5,6-chloromethyl-2',7'-dichlorodihydrofluorescein diacetate (DCF) assay. Formation of ROS in the epithelium was monitored and detected, by fluorescence, in intact bovine epithelial cells layers, from the different treatment groups. Figure 4a shows a molecule of non fluorescent (reduced and acetylated) DCF.
The di-acetyl ester of DCF is cell permeable and undergoes hydrolysis within cells, thus remain trapped within the cells. While the DCF is a poorly fluorescent molecule, upon its oxidation by ROS DCF is converted to a highly fluorescent compound that can be easily monitored. Figure $4 \mathrm{~b}$ demonstrates control lens epithelium after incubation of the intact lens for 12 days in culture. There is almost no fluorescence in the cells; minor fluorescence can be detected in the cells nuclei. On the other hand, glucose treated lenses epithelium show very high fluorescence, in the swollen epithelial cells (Figure 4c). DFO prevents the cytosolic oxidation, but high oxidation was observed within the nuclei (Figure $4 \mathrm{~d}$ ). $\mathrm{ZnDFO}$ provided better protection against oxidation than DFO or NAC. The epithelium from glucose-plus-ZnDFO treated lenses look like the controls (Figure 4e) Glucose and NAC treated lens epithelium showed swollen cells with less oxidation than in glucose-alone treated lens epithelium (Figure 4f).

In order to follow the effects of exposure to high glucose together

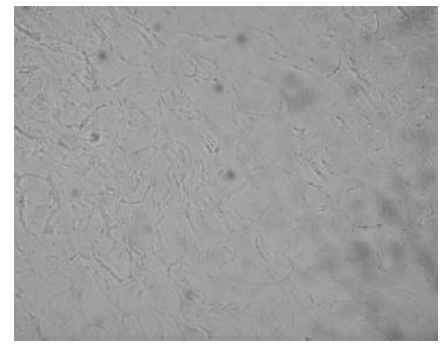

Figure 3d: Inverted microscope photograph of glucose and ZnDFO treated lens epithelium after 12 days incubation in organ culture conditions. (Magnification $x$ 100) glucose damage was reduced by ZnDFO treatment.

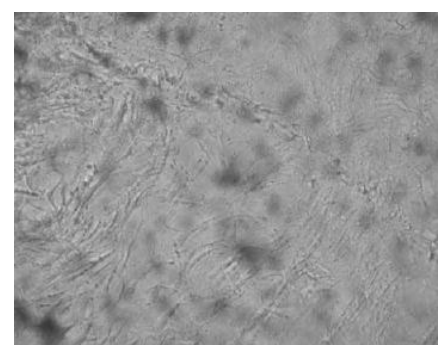

Figure 3e: Inverted microscope photograph of glucose and NAC treated lens epithelium after 12 days incubation in organ culture conditions. (Magnification $x 100)$ glucose damage was reduced by NAC treatment.<smiles>COC(=O)c1cc(C(F)(F)Cl)ccc1C1c2cc(Cl)c(OC(C)=O)cc2Oc2cc(OC(C)=O)c(Cl)cc21</smiles>

Figure 4a: Non-fluorescent DCF. 
Citation: Bormusov E, Dovrat A, Chevion M, Reznick AZ (2012) Antioxidants Prevention of Diabetic Damage in the Organ Culture Bovine Lenses. Metabolomics S1:004. doi:10.4172/2153-0769.S1-004

Page 4 of 7

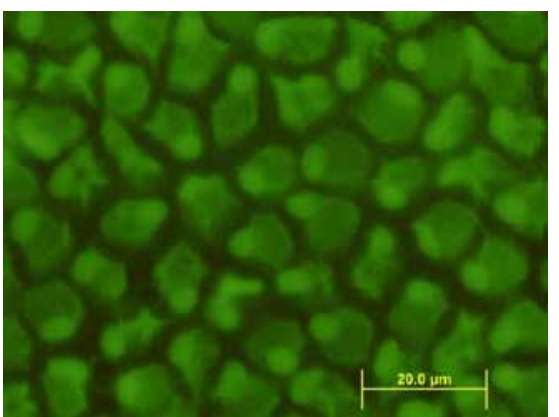

Figure 4b: Control lens epithelium after 12 days in organ culture conditions Note the low fluorescence of the epithelial cells.

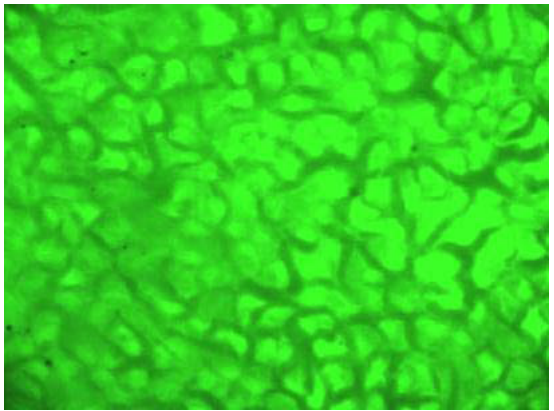

Figure 4c: Glucose treated lens epithelium after 12 days in organ culture conditions. Note the high fluoresce in the epithelial cells which indicate high oxidation.

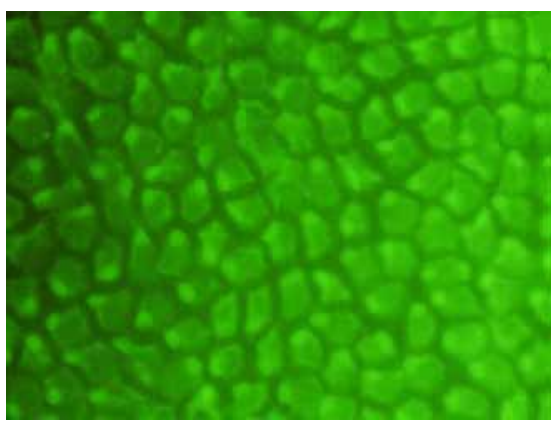

Figure 4d: Glucose and DFO treated lens epithelium after 12 days in organ culture conditions. DFO reduced the oxidation levels in the cells. Note that the main oxidation remained in the nuclei of the cells.

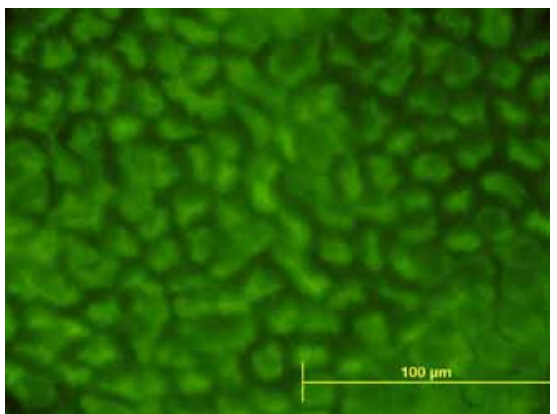

Figure 4e: Glucose and ZnDFO treated lens epithelium after 12 days in organ culture conditions. ZnDFO prevents the oxidation in the cells and the cells looks like the controls. with the antioxidants on the cells nuclei, the nuclei were stained with propidium iodide, an intercalating DNA staining fluorescent agent (Figure 5). Control lens epithelium show intact cells and intact nuclei (Figure 5a); glucose treated lens epithelium show swollen cells with broken nuclei (Figure 5b). DFO and NAC slightly reduced the damage to the cells nuclei (Figures $5 \mathrm{c}$ and $5 \mathrm{e}$ ), while $\mathrm{Zn} / \mathrm{DFO}$ totally prevent the damage. The epithelium from lenses treated with glucose in the presence of $\mathrm{ZnDFO}$ looks the same as the controls (Figure 5d).

The injurious effects of high glucose and the protective effects of the three antioxidants under study, on the profile of lens soluble proteins and their intactness were examined by $2 \mathrm{D}$ gel electrophoresis analysis (Figure 6). Protein profile of control lens soluble proteins is demonstrated in Figure 6a. The molecular weight of lens soluble proteins is below $32 \mathrm{kDa}$. Incubation of the lenses in the presence of $450 \mathrm{mg} \%$ glucose for 12 days reduce the amount of soluble proteins mainly at the basic area of the gel, the area of gamma crystalline (Figure

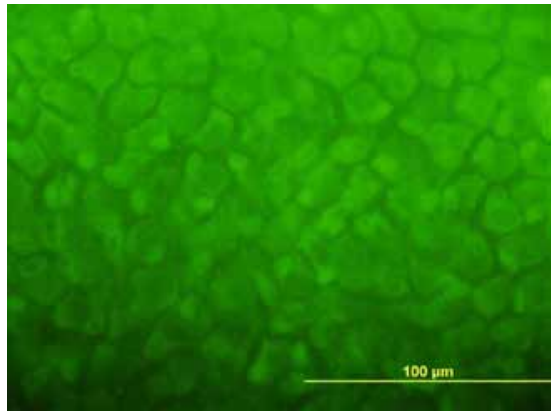

Figure 4f: Glucose and NAC treated lens epithelium after 12 days in organ culture conditions.

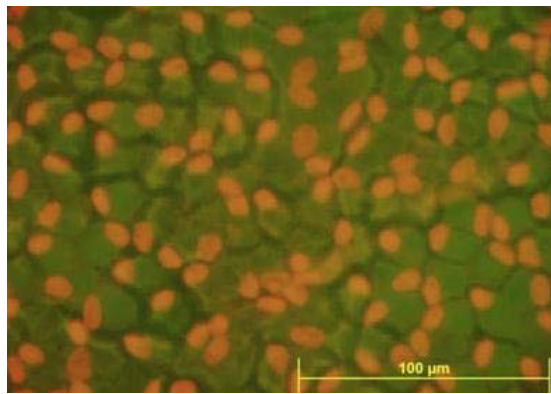

Figure 5a: Control lens epithelium after 12 days in organ culture conditions.

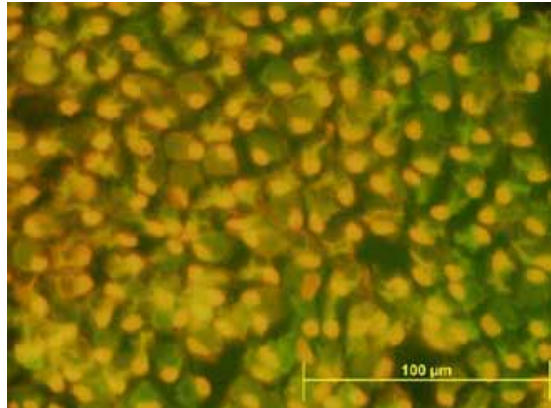

Figure 5b: Glucose treated lens epithelium after 12 days in organ culture conditions. 
Citation: Bormusov E, Dovrat A, Chevion M, Reznick AZ (2012) Antioxidants Prevention of Diabetic Damage in the Organ Culture Bovine Lenses. Metabolomics S1:004. doi:10.4172/2153-0769.S1-004

Page 5 of 7

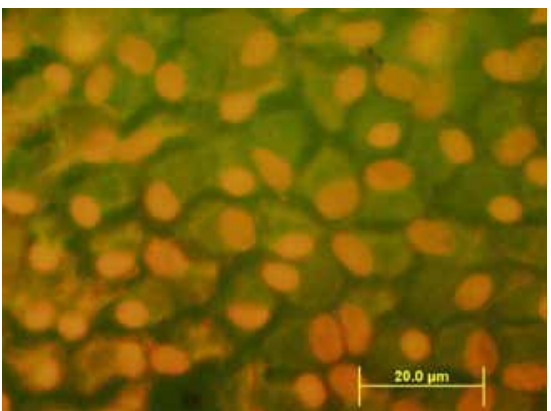

Figure 5c: Glucose and DFO treated lens epithelium after 12 days in organ culture conditions.

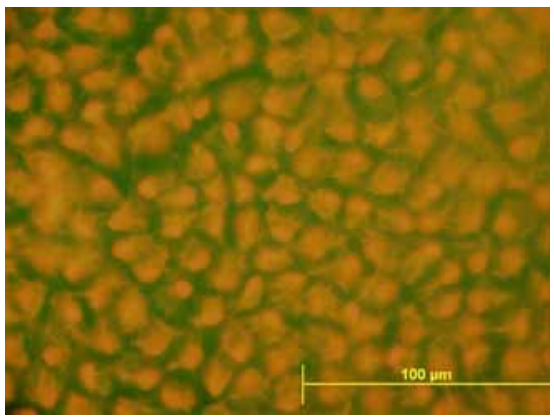

Figure 5d: Glucose and ZnDFO treated lens epithelium after 12 days in organ culture conditions.

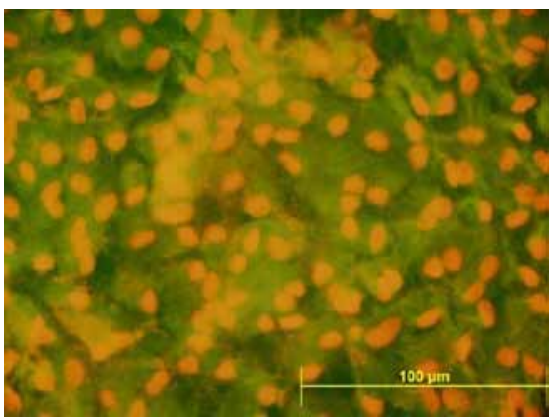

Figure 5e: Glucose and NAC treated lens epithelium after 12 days in organ culture conditions.

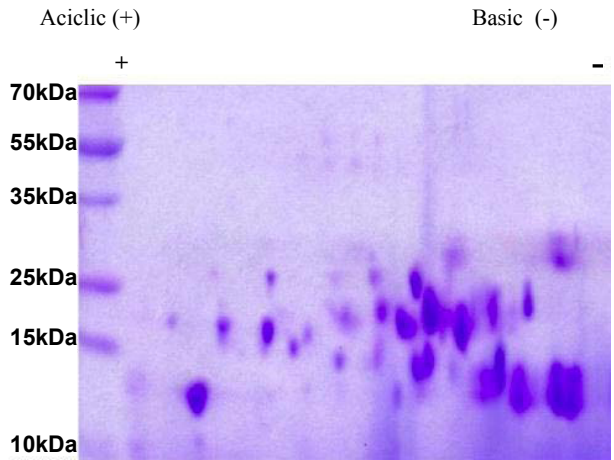

Figure 6a: Lens soluble protein profile (2D gel electrophoresis) of control lens after 12 day incubation in culture conditions. 6b) Incubation of the glucose treated lenses in the presence of DFO partially protect lens proteins as demonstrated in Figure 6c. Almost no protection to lens soluble proteins was provided in the presence of $\mathrm{ZnDFO}$ (Figure 6d). On the other hand full protection from glucose damage was provided in the presence of NAC. Lenses incubated in high glucose in the presence of NAC show the same soluble protein profile as the controls (Figure 6e).

We have demonstrated a role for oxidative damage in diabetic cataract formation. This is in accord with previous proposals [27-29]. Thus, the possible use of antioxidant agents in cataract prevention and treatment is highly appealing. Future investigations should consider

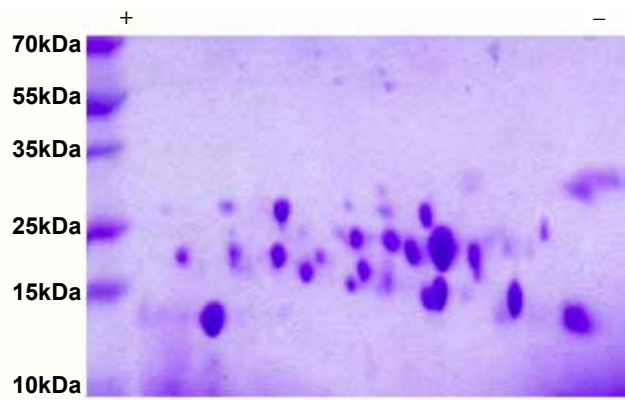

Figure 6 b: Lens soluble protein profile (2D gel electrophoresis) of glucose $(450 \mathrm{mg} \%)$ treated lens after 12 day incubation in culture conditions. Note the missing proteins mainly at the basic side of the gel, the area of gamma crystalline.

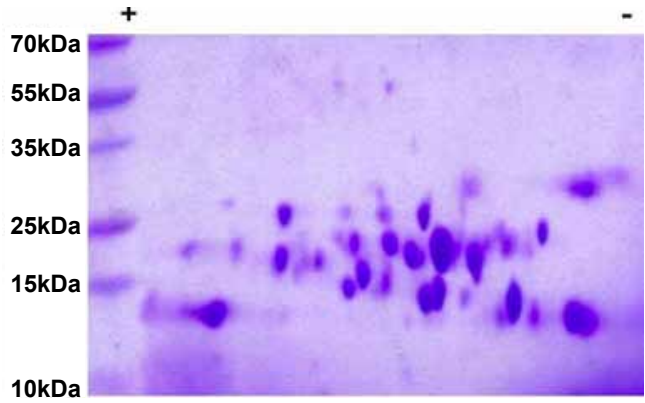

Figure 6c: Lens soluble protein profile (2D gel electrophoresis) of glucose $(450 \mathrm{mg} \%)+$ DFO treated lens after 12 day incubation in culture conditions. Note the missing proteins mainly at the basic side of the gel, the area of gamma crystalline.

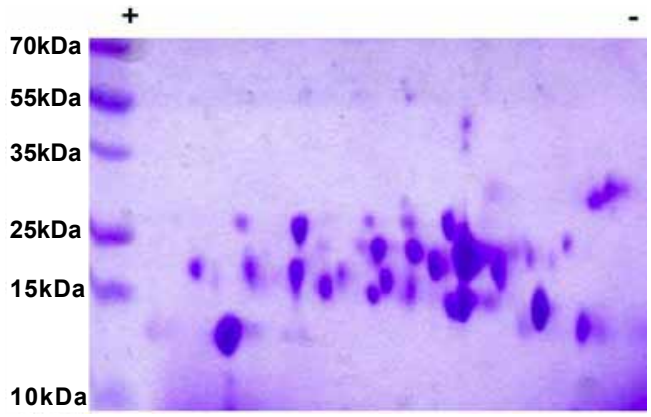

Figure 6d: Lens soluble protein profile (2D gel electrophoresis) of glucose $(450 \mathrm{mg} \%)+$ ZnDFO treated lens after 12 day incubation in culture conditions. Note the missing proteins mainly at the basic side of the gel, the area of gamma crystalline. 


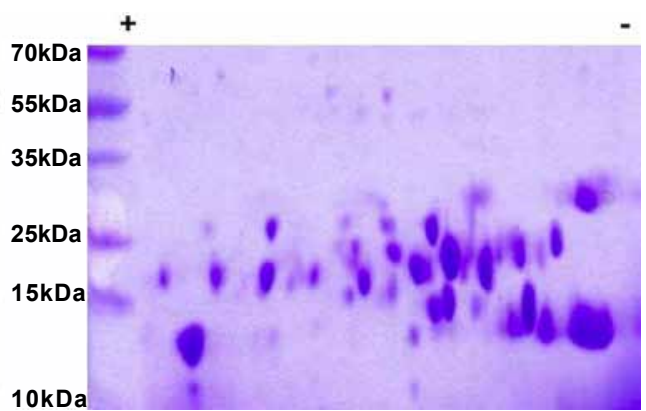

Figure 6e: Lens soluble protein profile (2D gel electrophoresis) of glucose $(450 \mathrm{mg} \%)+$ NAC treated lens after 12 day incubation in culture conditions. Note the protective effect of NAC on lens proteins.

this possibility in human subjects. It is likely that NAC and $\mathrm{Zn} / \mathrm{DFO}$ (rather than DFO alone) could provide a beneficial outcome.

In summary, we used a unique experimental lens culture system equipped with highly sensitive and reproducible detection monitoring instrumentation. When lenses were exposed to diabetic-like conditions, under incubation for 12 days in culture, an oxidative damage developed. This was in contrast to incubation of lenses under normo-glycemic medium, where the lenses remained clear and un-affected. When NAC was included in the incubation medium, no protein alterations were developed. Likewise $\mathrm{Zn} / \mathrm{DFO}$ provided complete protection against oxidative injury. DFO alone provided good, but limited, protection. We propose that these antioxidants, in particularly NAC and Zn/DFO should be considered as preventative treatment in diabetic patients, in order to delay or avoid, and possible treat ocular opacity.

Indeed, In vitro and in vivo studies have demonstrated that NAC, a thiol compound that is used clinically possesses not only antioxidant properties, but also anti-inflammatory and vasodilatory properties. NAC acts as a cysteine pro-drug and a GSH precursor [17]. It can reduce disulfide bonds in proteins [14,18], scavenge free radicals [15] and bind metals to form complexes [19]. This can explain our results, where the unusual protective effect of NAC on lenticular epithelium, was observed.

Further research is needed to examine whether within the intact animal NAC will prove as efficient. This is since pharmacokinetics studies have shown that NAC undergoes extensive first pass metabolism in the liver and kidneys resulting in low concentrations of 'free' NAC in the plasma $[30,31]$.

In earlier studies using the same model with non-diabetic lenses, we demonstrated that lens periphery is the most sensitive area first damaged by exposure to hyperbaric oxygen tension. While DFO proved only partially beneficial in salvaging the lens periphery from oxidative damage, $\mathrm{Zn} / \mathrm{DFO}$ led to complete protective benefit to the lens anterior pole, the part most relevant for sight preservation $[32,33]$. These observations were in accord with other publications demonstrating the beneficial effects of iron chelating agents against oxidative insult- induced tissue damage [7,8,34-37]. Typically, high dose of DFO mesylate (Desferal ${ }^{\mathbb{}}$ ) was administered, subcutaneously (10 mg/kg of body weight), for long term(up to 90 consecutive days), prevented cataractous changes in rats. The disadvantage of DFO in these studies stems from its limited tissue penetration [38,39]. Indeed, $\mathrm{Zn} / \mathrm{DFO}$ easily infiltrates into cells and tissues $[9,32,40]$ and is, thus, a better protective drug, as is evident also under conditions that simulate diabetes.
The unusual activity of $\mathrm{Zn} / \mathrm{DFO}$ is based on the combination of 'push and pull' mechanisms [10,41]. Desferrioxamine neutralized the redox activity of iron by binding labile ferric iron and 'pulling out' iron from its (low affinity) binding sites. Zinc ion is liberated from the complex during the exchange of the zinc with iron, and the 'free' zinc acts as a secondary antioxidant, 'pushing out' additional iron from binding sites, thus, inhibiting the catalysis of the formation of highly reactive ROS. The $\mathrm{Zn} / \mathrm{DFO}$ complex carries additional attributes which make it more effective - it infiltrates into cells, and eliminates the toxicity of free DFO.

It is a well accepted notion that oxygen and ROS play key roles in senile cataract formation. This view is supported by former observations demonstrating the rapid development of cataract under conditions of high oxygen load in humans treated by hyperbaric oxygen [42]. Oxygen is also believed to be one of the potential causative agents for the development of nuclear cataract following vitrectomy [43,44]. Under normal clinical circumstances the effects of oxygen-load accumulate over many years of exposure to relatively low oxygen loads. We have shown that increasing the partial pressure of oxygen, for a relatively short time causes a sharp increase in the damage to the lens.

\section{Conclusions}

Based on the independent effects of NAC and $\mathrm{Zn} / \mathrm{DFO}$, we propose to use their combination as a means of prevention and/or treatment of cataract, in diabetic patients.

This combination can be applied by topically drops or systemically by ingestion. It is anticipated that in both ways the antioxidants will get access to the lens through the posterior chamber of the aqueous humor. While the risk of using such a combination is very limited, further investigation is needed in order to crystallize, in detail, the efficiency and protocols of treatment of human subjects.

\section{References}

1. Harman D (1956) Aging: a theory based on free radical and radiation chemistry J Gerontol 11: 298-300.

2. Harman D (2003) The free radical theory of aging. Antioxid Redox Signal 5: 557-61.

3. Rosini M, Andrisano V, Bartolini M, Bolognesi ML, Hrelia P, et al. (2005) Rational approach to discover multipotent anti-Alzheimer drugs. J Med Chem 48: $360-363$

4. Marsili S, Salganik RI, Albright CD, Freel CD, Johnsen S, et al. (2004) Cataract formation in a strain of rats selected for high oxidative stress. Exp Eye Res 79 595-612.

5. Giblin FJ, McCready JP (1983) The effect of inhibition of glutathione reductase on the detoxification of $\mathrm{H} 2 \mathrm{O} 2$ by rabbit lens. Invest Ophthalmol Vis Sci 24 113-118.

6. Garner B, Davies MJ, Truscott RJ (2000) Formation of hydroxyl radicals in the human lens is related to the severity of nuclear cataract. Exp Eye Res: 70: $81-88$

7. Avunduk AM, Yardimci S, Avunduk MC, Kurnaz L (1999) Cataractous changes in rat lens following cigarette smoke exposure is prevented by parenteral deferoxamine therapy. Arch Ophthalmol 117: 1368-1372.

8. Reddan J, Sevilla M, Giblin F, Padgaonkar V, Dziedzic D, et al. (1992) Tempo and deferoxamine protect cultured rabbit lens epithelial cells from $\mathrm{H} 2 \mathrm{O} 2$ insult: insight into the mechanism of $\mathrm{H} 2 \mathrm{O} 2-$ induced injury. Lens Eye Toxic Res 9 : 385-393.

9. Chevion M, Chuang L, Golenser J (1995) Effects of zinc-desferrioxamine on Plasmodium falciparum in culture. Antimicrob Agents Chemother 39: $1902-$ 1905.

10. Karck M, Tanaka S, Berenshtein E, Sturm C, Haverich A, et al. (2001) The 
Citation: Bormusov E, Dovrat A, Chevion M, Reznick AZ (2012) Antioxidants Prevention of Diabetic Damage in the Organ Culture Bovine Lenses. Metabolomics S1:004. doi:10.4172/2153-0769.S1-004

push-and-pull mechanism to scavenge redox-active transition metals: a nove concept in myocardial protection. J Thorac Cardiovasc Surg 121: 1169-78.

11. Banin E, Berenshtein E, Kitrossky N, Pe'er J, Chevion M (2000) Galliumdesferrioxamine protects the cat retina against injury after ischemia and reperfusion. Free Radic Biol Med 28: 315-323.

12. Chevion M, Berenshtein E (1997) United States Patent and Trademark Office, assignee. Gallium complexes for the treatment of free radical-induced diseases. USA patent $5,618,838$.

13. Banin E, Lozinski A, Brady KM, Berenshtein E, Butterfield PW, et al. (2008) The potential of desferrioxamine-gallium as an anti- Pseudomonas therapeutic agent. Proc Natl Acad Sci U S A 105: 16761-6.

14. Ziment I (1988) Acetylcysteine: a drug that is much more than a mucokinetic. Biomed Pharmacother 42: 513-519.

15. Aruoma OI, Halliwell B, Hoey BM, Butler J (1989) The antioxidant action of $\mathrm{N}$-acetylcysteine: its reaction with hydrogen peroxide, hydroxyl radical, superoxide, and hypochlorous acid. Free Radic Biol Med 6: 593-7.

16. Atkuri KR, Mantovani JJ, Herzenberg LA, Herzenberg LA (2007) $\mathrm{N}$-Acetylcysteine--a safe antidote for cysteine/glutathione deficiency. Curr Opin Pharmacol 7: 355-359.

17. Zafarullah M, Li WQ, Sylvester J, Ahmad M (2003) Molecular mechanisms of $\mathrm{N}$-acetylcysteine actions. Cell Mol Life Sci 60: 6-20.

18. Harada D, Anraku M, Fukuda H, Naito S, Harada K, et al. (2004) Kinetic studies of covalent binding between $\mathrm{N}$-acetyl-L-cysteine and human serum albumin through a mixed-disulfide using an N-methylpyridinium polymer-based column. Drug Metab Pharmacokinet 19: 297-302.

19. Koh AS, Simmons-Willis TA, Pritchard JB, GrassI SM, Ballatori N (2002) Identification of a mechanism by which the methylmercury antidotes $\mathrm{N}$-acetylcysteine and dimercaptopropanesulfonate enhance urinary metal excretion: transport by the renal organic anion transporter-1. Mol Pharmacol 62: 921-6.

20. Sivak JG, Yoshimura M, Weerheim J, Dovrat A (1990) Effect of hydrogen peroxide, DL-propranolol, and prednisone on bovine lens optical function in culture. Invest Ophthalmol Vis Sci 31: 954-63.

21. Dovrat A, Sivak JG (2005) Long-term lens organ culture system with a method for monitoring lens optical quality. Photochem Photobiol 81: 502-5.

22. Bloemendal H, Van de gaer K, Benedetti EL, Dunia I, Steely HT (1997) Towards a human crystallin map. Two-dimensional gel electrophoresis and computer analysis of water-soluble crystallins from normal and cataractous human lenses. Ophthalmic Res 29: 177-90.

23. Lowry OH, Rosebrough NJ, Farr AL, Randall RJ (1951) Protein measurement with the Folin phenol reagent. J Biol Chem 193: 265-75.

24. Di Benedetto A, Aragona P, Romano G, Romeo G, Di Cesare E, et al. (1999) Age and metabolic control influence lens opacity in type I, insulin-dependent diabetic patients. J Diabetes Complications 13: 159-62.

25. Freel CD, Gilliland KO, Wesley Lane C, Giblin FJ, Joseph Costello M (2002) Fourier analysis of cytoplasmic texture in nuclear fiber cells from transparent and cataractous human and animal lenses. Exp Eye Res 74: 689-702.

26. Tkachov SI, Lautenschlager C, Ehrich D, Struck HG (2006) Changes in the lens epithelium with respect to cataractogenesis: light microscopic and Scheimpflug densitometric analysis of the cataractous and the clear lens of diabetics and non-diabetics. Graefes Arch Clin Exp Ophthalmol 244: 596-602.

27. Lou MF (2003) Redox regulation in the lens. Prog Retin Eye Res 22: 657-682.

28. Shin DM, Jeon JH, Kim CW, Cho SY, Kwon JC, et al. (2004) Cell type-specific activation of intracellular transglutaminase 2 by oxidative stress or ultraviolet irradiation: implications of transglutaminase 2 in age-related cataractogenesis. J Biol Chem 279: 15032-15039.

29. Tarwadi K, Agte V (2004) Linkages of antioxidant, micronutrient, and socioeconomic status with the degree of oxidative stress and lens opacity in Indian cataract patients. Nutrition 20: 261-267.

30. Holdiness MR(1991) Clinical pharmacokinetics of $\mathrm{N}$-acetylcysteine. Clin Pharmacokinet 20: 123-134.

31. Cotgreave IA (1997) N-acetylcysteine: pharmacological considerations and experimental and clinical applications. Adv Pharmacol 38: 205-27.
32. Schaal S, Beiran I, Bormusov E, Chevion M, Dovrat A (2007) Zincdesferrioxamine reduces damage to lenses exposed to hyperbaric oxygen and has an ameliorative effect on catalase and $\mathrm{Na}, \mathrm{K}-\mathrm{ATPase}$ activities. Exp Eye Res 84: 455-63.

33. Schaal S, Beiran I, Rozner H, Rubinstein I, Chevion M, et al. (2007) Desferrioxamine and zinc-desferrioxamine reduce lens oxidative damage. Exp Eye Res 84: 561-8.

34. Goralska M, Holley BL, McGahan MC (2003) Identification of a mechanism by which lens epithelial cells limit accumulation of overexpressed ferritin $\mathrm{H}$-chain. J Biol Chem 278: 42920-6.

35. Argirova M, Kleine-Reidick M, Breipohl W (2004) Redox status of the eye lens: a regional study. Cell Biochem Biophys 41: 381-90.

36. Banin E, Morad Y, Berenshtein E, Obolensky A, Yahalom C, et al. (2003) Injury induced by chemical warfare agents: characterization and treatment of ocular tissues exposed to nitrogen mustard. Invest Ophthalmol Vis Sci 44: 2966-72.

37. Morad Y, Banin E, Averbukh E, Berenshtein E, Obolensky A, et al. (2005) Treatment of ocular tissues exposed to nitrogen mustard: beneficial effect of zinc desferrioxamine combined with steroids. Invest Ophthalmol Vis Sci 46 : 1640-6.

38. Samuni Y, Coffin D, DeLuca AM, DeGraff WG, Venson DJ, et al. (1999) The use of $\mathrm{Zn}$-desferrioxamine for radioprotection in mice, tissue culture, and isolated DNA. Cancer Res 59: 405-9.

39. Cable H, Lloyd JB (1999) Cellular uptake and release of two contrasting iron chelators. J Pharm Pharmacol 51: 131-4.

40. Nagler R, Marmary Y, Golan E, Chevion M (1998) Novel protection strategy against $X$-ray-induced damage to salivary glands. Radiat Res 149: 271-6.

41. Chevion M (1991) Protection against free radical-induced and transition metalmediated damage: the use of "pull" and "push "mechanisms. Free Radic Res Commun 12: 691-6.

42. Palmquist BM, Philipson B, Barr PO (1984) Nuclear cataract and myopia during hyperbaric oxygen therapy. Br J Ophthalmol 68: 113-7.

43. Barbazetto IA, Liang J, Chang S, Zheng L, Spector A, et al. (2004) Oxygen tension in the rabbit lens and vitreous before and after vitrectomy. Exp Eye Res 78: 917-24.

44. Harocopos GJ, Shui YB, McKinnon M, Holekamp NM, Gordon MO, et al. (2004) Importance of vitreous liquefaction in age-related cataract. Invest Ophthalmol Vis Sci 45: 77-85. 